OPEN ACCESS

Edited by:

Guy Smagghe,

Ghent University, Belgium

Reviewed by:

Hao Guo,

Chinese Academy of Sciences (CAS),

China

Qili Feng,

South China Normal University, China

Luc Swevers,

National Centre of Scientific Research

"Demokritos", Greece

${ }^{*}$ Correspondence:

Wei Sun

sunwei077@cqu.edu.cn

Specialty section:

This article was submitted to

Invertebrate Physiology,

a section of the journal

Frontiers in Physiology

Received: 07 November 2019

Accepted: 24 April 2020

Published: 09 June 2020

Citation:

Duan H, Yang $X$, Bu Z, Li X,

Zhang $Z$ and Sun W (2020) Identification and Characterization of Genes Involved in Ecdysteroid Esterification Pathway Contributing to the High 20-Hydroxyecdysone

Resistance of Helicoverpa armigera.

Front. Physiol. 11:508

doi: 10.3389/fphys.2020.00508

\section{Identification and Characterization of Genes Involved in Ecdysteroid Esterification Pathway Contributing to the High 20-Hydroxyecdysone Resistance of Helicoverpa armigera}

\author{
Hengtong Duan, Xin Yang, Zhanyao Bu, Xinyue Li, Ze Zhang and Wei Sun* \\ Laboratory of Evolutionary and Functional Genomics, School of Life Sciences, Chongqing University, Chongqing, China
}

20-Hydroxyecdysone (20E), the most important regulator for insect development, is also a major component in phytoecdysteroids in plants. Therefore, this plant-derived hormone is considered as a potential natural product for use in pest management. However, some insects show high resistance to it, and the molecular mechanism of their resistance is still unclear. In this study, we find that the cotton bollworm Helicoverpa armigera larvae show high tolerance to artificial foods containing up to $50 \mu \mathrm{g} 20 \mathrm{E}$ without any detrimental effects on growth and development. High performance liquid chromatography analysis indicates that high efficiency to transform the ingested 20E through an ecdysteroid esterification pathway may contribute to the resistance. Furthermore, comparative transcriptome analysis of the larvae's midgut after 20E treatment identifies two genes (long-chain-fatty-acid-CoA ligase, Long-FACL; sterol O-acyltransferase, SATF) involved in the pathway. Transcriptome and real-time PCR show the Long-FACL gene can be significantly induced by $20 \mathrm{E}$, and this induction is only detected in the midgut. However, 20E has no effect on the transcript of the SATF gene. Moreover, the heterologously expressed protein of the SATF gene shows the ecdysteroid-22-O-acyltransferase activity that requires fatty acyl-CoA, which is produced by Long-FACL. Taken together, our results identify and demonstrate the genes involved in the ecdysteroid esterification pathway conferring high resistance to $20 \mathrm{E}$ in the cotton bollworm, $H$. armigera.

Keywords: phytoecdysteroids, detoxification, ecdysteroid esterification pathway, transcriptome, cotton bollworm

\section{INTRODUCTION}

Ecdysteroids are one of the most important hormones in insects. They are recognized as essential regulators controlling molting, metamorphosis, and reproduction in insects. Since the isolation of ecdysone from silkworm pupae in 1954, ecdysteroid analogs called phytoecdysteroids were also discovered in plant species (Butenandt and Karlson, 1954; Nakanishi et al., 1966; Lafont and Horn, 1989; Dinan, 2001). Many plants contain a relatively high concentration of ecdysteroids (up to $3 \%$ of their dry weight) (Dinan, 2001). Moreover, the major form of phytoecdysteroids in plants is 20-hydroxyecdysone (20E), which is also the most biologically active ecdysteroid in insects 
(Lafont and Horn, 1989). Therefore, phytoecdysteroids are considered to be a defensive system to protect plants against phytophagous invertebrates (Adler and Grebenok, 1999; Mithöfer and Boland, 2012). In this regard, phytoecdysteroids were indeed demonstrated to be antifeedants toward some insects, such as the dark-winged fungus gnat (Bradysia impatiens) and polyphagous Japanese beetle Popillia japonica (Schmelz et al., 2002; Jurenka et al., 2017). More importantly, phytoecdysteroids could execute direct toxicity to insects. Orally ingested low levels of phytoecdysteroids could disrupt normal development, reduce fecundity, or induce death of some lepidopteran insects, such as the sweet-potato whitefly Bemisia tabaci and the persea mite Oligonychus perseae (Kubo et al., 1981, 1983; Tanaka and Naya, 1995; Blackford and Dinan, 1997a,b; Mondy et al., 1997). Consequently, phytoecdysteroids have been suggested to be a good candidate for pest management (Soriano et al., 2004; Aly et al., 2011; Jurenka et al., 2017; Chaubey, 2018).

However, previous studies have reported that a number of pests, especially certain noctuid insects, showed high resistance to phytoecdysteroids. Some Helicoverpa species (Helicoverpa virescens and Helicoverpa zea) can tolerate high concentrations of $20 \mathrm{E}$ in their diet without any detrimental effects (Kubo et al., 1981). Blackford et al. (1996, 1997) also found that exogenous application of $20 \mathrm{E}$ could not affect the normal growth and development of Spodoptera littoralis and Lacanobia oleracea (Blackford et al., 1996; Blackford and Dinan, 1997b). Furthermore, in those noctuid insects, the high resistance is mainly due to the effective conversion of the ingested ecdysteroids into 22-long-chain-fatty-acyl esters in the gut (Kubo et al., 1987; Robinson et al., 1987; Zhang and Kubo, 1993; Blackford et al., 1997). The ecdysteroid-22-acyl esters show over 100 times less activity than 20E (Zhang and Kubo, 1992b). In noctuid insects, the conversion is considered to be an important pathway of detoxification of ingested exogenous ecdysteroids. This esterification process is mediated by ecdysteroid-22-Oacyltransferase, which biochemical characteristics have been described in previous studies (Zhang and Kubo, 1992a,b; Kubo et al., 1994). However, the gene encoding ecdysteroid-22-Oacyltransferase is still unclear.

The cotton bollworm, Helicoverpa armigera (Lepidoptera: Noctuidae), is the most significant and impactful pest in agriculture. The pest is a typical polyphagous insect, which feeds on at least 180 plant species from $>30$ families, causing serious crops damage and economic losses (Martin et al., 2005). The wide feeding spectrum of the cotton bollworm indicates that some efficient processes must be involved in detoxifying the plant-derived substances. Similar to other noctuid insects, the cotton bollworm can also show resistance to $20 \mathrm{E}$ through the ecdysteroids esterification pathway (Robinson et al., 1987). In this study, we used the cotton bollworm as a model, and performed transcriptomic analysis to measure the effect of exogenous $20 \mathrm{E}$ on $H$. armigera. Thereafter, we identified and characterized the function of the genes involved in the ecdysteroids esterification pathway. The data shown in this study will help us to understand how insects adapt to and conquer the secondary metabolites of their host plants.

\section{MATERIALS AND METHODS}

\section{Insects Culture}

Eggs of $H$. armigera and artificial diets were purchased from Keyun Bioinsecticide Research and Development Center of the Chinese Academy of Sciences Institute of Zoology (Henan, China). The larvae were reared on an artificial diet at $27^{\circ} \mathrm{C}$ under a 12/12-h light/dark photoperiod.

\section{Insect Treatment}

The artificial diet was cut into small pieces of diet blocks (about $25 \mathrm{mg} /$ block), and then the blocks were mixed with different amount of 20E (20 $\mu \mathrm{g} / \mathrm{block} ; 50 \mu \mathrm{g} / \mathrm{block}$; Sigma-Aldrich, St. Louis, MO). The same size diet blocks with $20 \%$ ethanol were used as the control. For ingestion experiments, the day 1 larvae of the sixth instar cotton bollworm were firstly starved for $6 \mathrm{~h}$ to accelerate feeding rate, and then each larva was fed on one mixed diet block. All the larvae could completely eat the diet block within $1 \mathrm{~h}$. Then the larva was transferred to a normal diet block (about $0.5 \mathrm{~g} / \mathrm{block}$ ). Larvae and feces were weighted at different time points after treatment. In addition, the remaining foods at each measured time point were also weighted, and then were replaced by a new known weight of an artificial diet block.

According to previous studies, we calculated some nutritional parameters to measure the effects of ingested 20E on larvae: weight gain, amount of ingested food, digestibility, efficiency of conversion of digested food (ECD), and efficiency of conversion of ingested food (ECI). The calculation formulas of those parameters are the same as in the previous study (Blackford et al., 1996).

\section{Dissection and Extraction}

For each time point, a hemolymph of each larva was collected by bleeding the proleg. Methanol was added to the hemolymph $(100 \mu \mathrm{L})$ from the larva to a final volume of $1 \mathrm{~mL}$. The feces and fat body dissected from each larva were extracted overnight with $2 \mathrm{~mL}$ of methanol. The supernatant was collected and evaporated. The dried samples were dissolved in ethanol and stored at $-20^{\circ} \mathrm{C}$.

\section{Total RNA Isolation and RNA-seq}

After $20 \mathrm{E}$ or $20 \%$ ethanol treatment at different time points, the midgut, head, fatbody, and epidermis were dissected on ice and immediately frozen and stored in liquid nitrogen, respectively. Every tissue sample was collected from five larvae. Previous studies have shown that the larval midgut was the main tissue to detoxify the exogenous 20E. Therefore, the midgut was collected at $3 \mathrm{~h}$ after hormone treatment to perform transcriptomic analysis.

All dissected tissues were grinded in liquid nitrogen to powders. Total RNA was extracted by the Ultrapure RNA kit (Beijing CoWin Biotech, Beijing, China) and treated with DNase I (Takara Bio, Shiga, Japan) to remove the genomic DNA contamination. The RNA was quantified by the UV spectrophotometer.

For transcriptome sequencing, the RNA samples were sent to the Biomarker Technologies Corporation (Beijing, China) 
for cDNA library construction and RNA-seq by Illumina HiSeq X Ten (Illumina, San Diego, CA, United States) with 125 bp paired-end reads according to the manufacturer's instructions. The raw data of RNA-seq has been submitted to NCBI (SRA accession: PRJNA588578).

\section{Analyses on RNA-seq Data}

The transcriptomic reads data were mapped to the $H$. armigera reference genome (GenBank assembly accession: GCA_002156985.1) using HISAT2 software and analyzed using StringTie (Pertea et al., 2016). HTSEQ software was used to calculate read counts of each gene (Anders et al., 2015). The expression levels of genes were estimated using TBTools and normalized using the TPM (Transcripts per million; Chen et al., 2018). DEseq2 R package was performed to identify differently expressed genes (DEGs), and genes with Log2-fold-expressionchange greater than 2 , and corrected $P$-values less than 0.05 were considered to be differentially expressed (Love et al., 2014).

Gene ontology term $(\mathrm{GO})^{1}$ annotations were assigned by Blast2GO software (b2g4pipe_v2.5; Götz et al., 2008). The KEGG (Kyoto Encyclopedia of Genes and Genomes) annotations were performed using Blastall software against the KEGG database ${ }^{2}$ (Kanehisa and Goto, 2000). KEGG and GO enrichment analyses of DEGs were performed on an online platform ${ }^{3}$.

\section{Real-Time PCR}

For each treatment shown above, $1 \mu \mathrm{g}$ RNA was reverse transcribed to the first strand of cDNA by the EasyScript one-step gDNA removal and cDNA synthesis SuperMix kit (TransGen Biotech). The specific primers were designed and used in the quantitative real-time PCR analysis (Supplementary Table S1). The quantitative real-time PCR was performed using a realtime PCR detection system (CFX96, Bio-Rad, Hercules, CA) with a QuantiNova SYBR Green PCR Kit (Qiagen). The PCR was carried out as follows: 2 min at $95^{\circ} \mathrm{C}$, followed by 40 cycles of $5 \mathrm{~s}$ at $95^{\circ} \mathrm{C}$ and $15 \mathrm{~s}$ at $60^{\circ} \mathrm{C}$. The cotton bollworm ribosomal protein L3 gene (GenBank ID no. XM_021328338) was used as the reference gene.

\section{Eukaryotic Expression of the Candidate Genes}

The cDNA of the candidate genes was cloned into the pMK33/pMtHy-based vector between the metallothionein promoter (pMT) and the SV40 polyadenylation. The constructed plasmid was confirmed by sequencing. Then the recombinant plasmids were transfected into a Drosophila S2 cell line using an effectene transfection reagent (Qiagen). The transfected method was used according to the manufacturer's instructions. Twenty-four hours after transfection, a final concentration of $500 \mu \mathrm{M}$ copper sulfate was added to induce the expression of the targeted genes for $18 \mathrm{~h}$. Then the cells were homogenized in $50 \mathrm{mM}$ Tris- $\mathrm{HCl}$ (pH 7.4) containing 1 mM EDTA. The protein was quantified using the BCA protein assay.

\footnotetext{
${ }^{1}$ http://www.geneontology.org/

${ }^{2}$ http://www.genome.jp/kegg/

${ }^{3}$ http://www.omicshare.com/tools/
}

To examine whether the gene was expressed, western blotting analysis was performed. The Flag antibody (at a dilution of 1:5000; Beyotime, Shanghai, China) was used to detect the recombinant protein. The procedure of the western blotting was described previously (Sun et al., 2012).

The DNA sequences of candidate genes obtained in this study were deposited in GenBank (Genbank ID, SATF: MN688744; Cyp6B2: MN688745; Long-FACL: MN688746).

\section{Enzyme Assay, Ecdysteroid Extraction, and High Performance Liquid Chromatography Analysis}

To measure the enzyme activity, $500 \mu \mathrm{L}$ reaction, containing $0.1 \mathrm{mg}$ crude recombinant enzyme solution, $100 \mu \mathrm{g} 20 \mathrm{E}$, $0.06 \mathrm{mM}$ oleoyl-CoA which served as acyl-group donor, and $10 \mathrm{mM}$ phosphate buffer (PH 7.0), was incubated for $30 \mathrm{~min}$ at $20^{\circ} \mathrm{C}$ (Kubo et al., 1994). The reaction was quenched with $4.5 \mathrm{ml}$ ice-cold $100 \%$ methanol, and proteins were removed by centrifugation. The supernatant was collected and evaporated. The dried samples were dissolved in ethanol and stored at $-20^{\circ} \mathrm{C}$. Ecdysteroids were analyzed by reversed-phase high performance liquid chromatography (HPLC) using a C18 Nova-Pak cartridge $(4.6 \times 250 \mathrm{~mm}$; Waters Associates) which was performed on a Hitachi Primaide HPLC system (Hitachi, Japan) linked to a Dual $\lambda$ Absorbance UV detector set at $245 \mathrm{~nm}$. The mobile phase consisted of methanol-water $(93: 7, \mathrm{v} / \mathrm{v})$, and the elution rate was set at $1 \mathrm{~mL} / \mathrm{min}$.

In addition, the concentration of $20 \mathrm{E}$ and its derivates was calculated by comparing the peak area of the analyte in the sample with the peak area of the standard of a known concentration.

\section{Mass Spectrum}

Mass spectrometry was performed to identify the metabolites of 20E. Mass spectrometry was conducted by a Finnigan TSQ Quantum Ultra AM spectrometer (Thermo Electron Corp., San Jose, CA) equipped with an ESI source in the positive ion mode. The spray voltage was fixed at $3.0 \mathrm{kV}$ and the capillary temperature at $200^{\circ} \mathrm{C}$. The collision energy was fixed at $20 \mathrm{eV}$. Nitrogen was used as the sheath (20 psi) and auxiliary (8 psi) gas.

\section{Statistical Analysis}

All the experiments shown above were independently repeated three times. All statistical analyses in this study were performed in the statistical R package (Student's $t$ test; Mann-Whitney $U$ test).

\section{RESULTS}

\section{Ingested 20E Has No Effect on the Growth and Development of the Cotton Bollworm}

To ascertain whether exogenous $20 \mathrm{E}$ may affect growth and development, we mixed 20E with artificial diet and fed the 1day-old cotton bollworm larvae. Here, we utilized two different concentrations of $20 \mathrm{E}(20 \mu \mathrm{g} / \mathrm{larva}$ and $50 \mu \mathrm{g} / \mathrm{larva})$ which are almost 15 and 36 folds higher than the top peak of $20 \mathrm{E}$ 
titer during the metamorphosis stage, respectively (Kang et al., 2019). The artificial diet containing high concentrations of $20 \mathrm{E}$ (up to $50 \mu \mathrm{g} /$ larva) were applied. All the tested larvae could quickly consume the treated diet within $1 \mathrm{~h}$ and then were fed on a normal artificial diet. We found the high dosage of the hormone did not affect the growth and development of cotton bollworm larvae. The larvae treated with $20 \mathrm{E}$ gained weight and pupated at the same time as the controls, producing normal pupae with a similar weight and emerged as perfect adults (Figures 1A,B). Meanwhile, we also measured some nutrition parameters to further assess the influence of the ingested 20E. Larvae treated with $20 \mathrm{E}$ could consume a similar amount of artificial diet to the control (Figures 1C,D), and the conversion of ingested (ECI) and digested (ECD) food into the body also had no significant difference among the three tested groups
(Figures 1E,F). Thus, the cotton bollworm larvae has a high tolerance to the exogenous ingested $20 \mathrm{E}$.

To examine the metabolic fate of ingested $20 \mathrm{E}$ in larvae of the cotton bollworm, feces was collected from larvae fed on 20E (20 $\mu \mathrm{g} /$ larva) after treatment. Three hours after treatment, compared with the control, we found three additional peaks in the feces of the 20E fed larvae. According to the elution time of the standard sample, the first additional peak (FAP, retention time: about $8 \mathrm{~min}$ ) is 20E (Figure 2A). Furthermore, we used mass spectrum to identify the second additional peak (SAP, retention time: about $15 \mathrm{~min}$ ) and the third additional peak (TAP, retention time: about $21 \mathrm{~min}$ ). Significant fragmentation peaks of the SAP were observed at $m / z 745,727,709,463$, 445,427 , and 301 . For the TAP, the main ion peaks were at $m / z$ 747, 729, 711, 463, 445, 427, and 301 (Supplementary
A
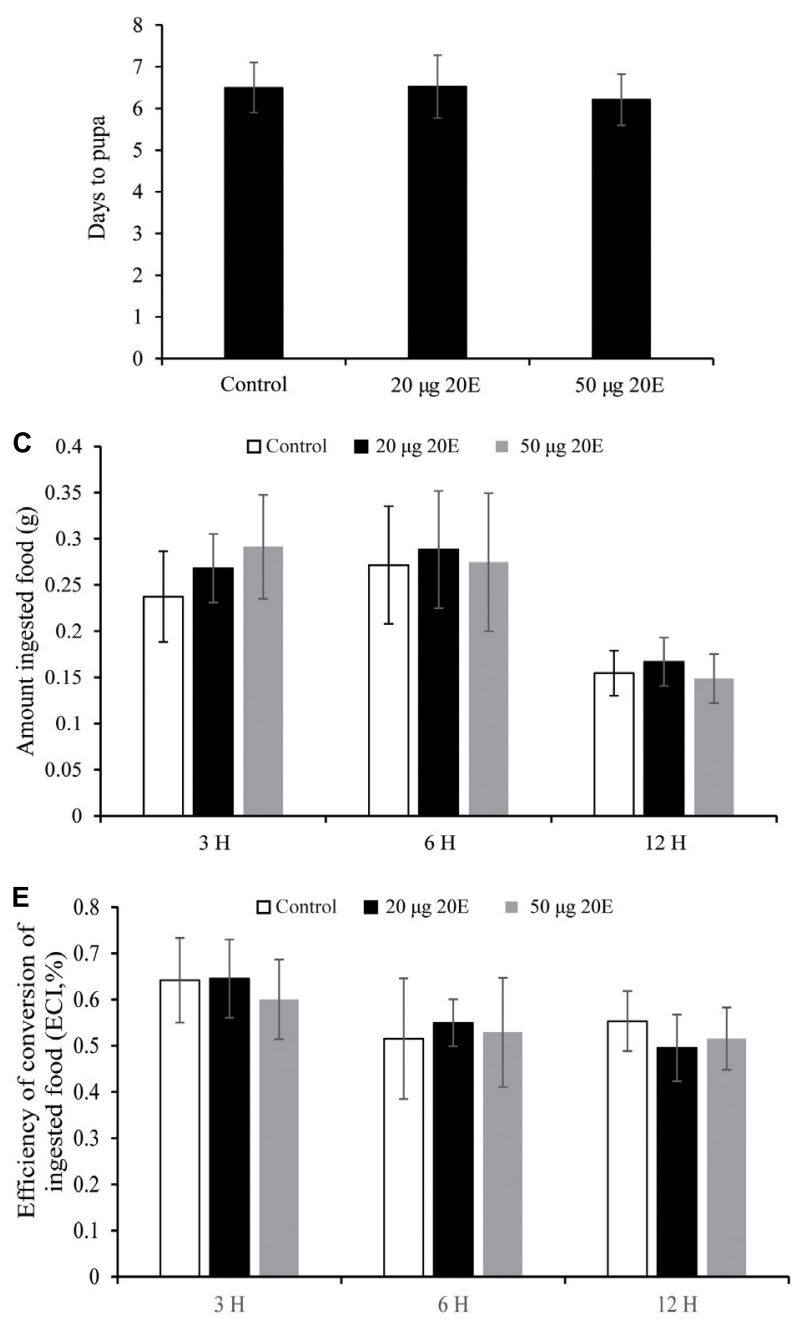

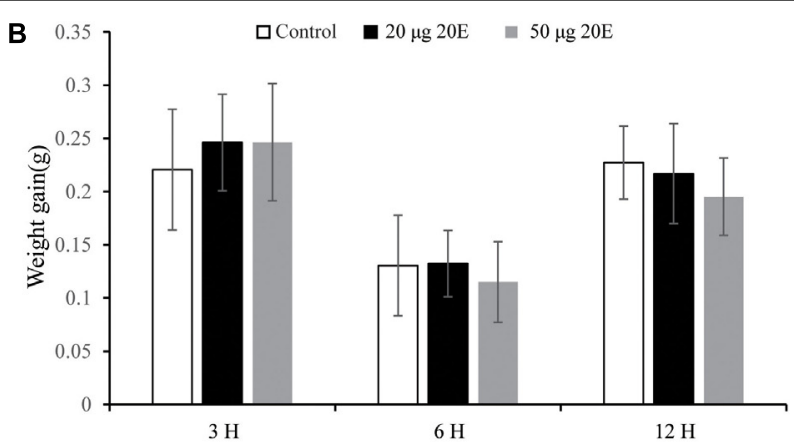

D

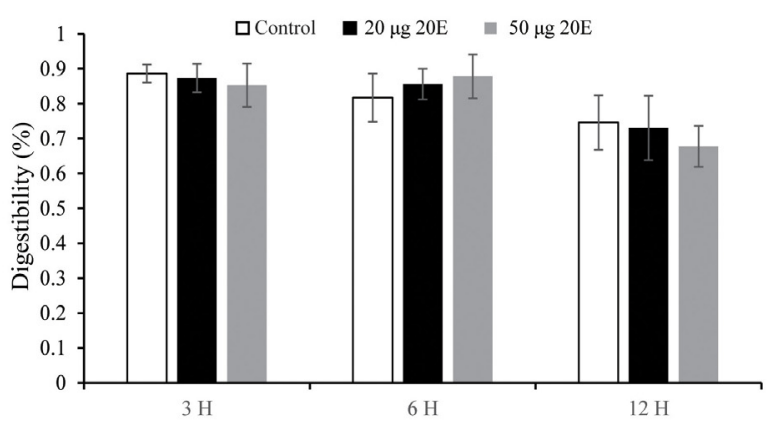

$\mathbf{F}$

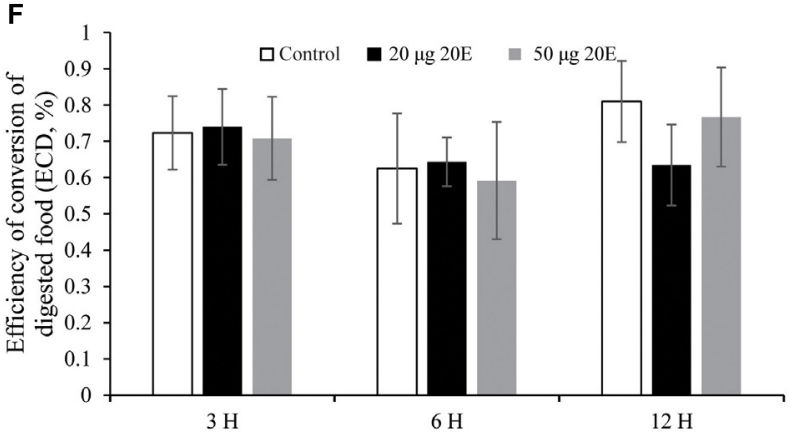

FIGURE 1 | Effects of the ingested 20E on the growth and development of the cotton bollworm larvae. (A) The time to pupation of the larvae reared on food treated with control or different concentrations of 20E. (B) The weight gain of the larvae reared on food treated with control or 20E. (C) The average amount of ingested food by the control or 20E treated larvae. (D) The digestibility of the larvae treated with control or 20E; (E) The efficiency of conversion of ingested food (ECI) of the larvae treated with control or 20E. (F) The efficiency of conversion of digested food (ECD) of the larvae treated with control or $20 \mathrm{E}$. Mann-Whitney $U$ test was used to do the statistic analysis. 

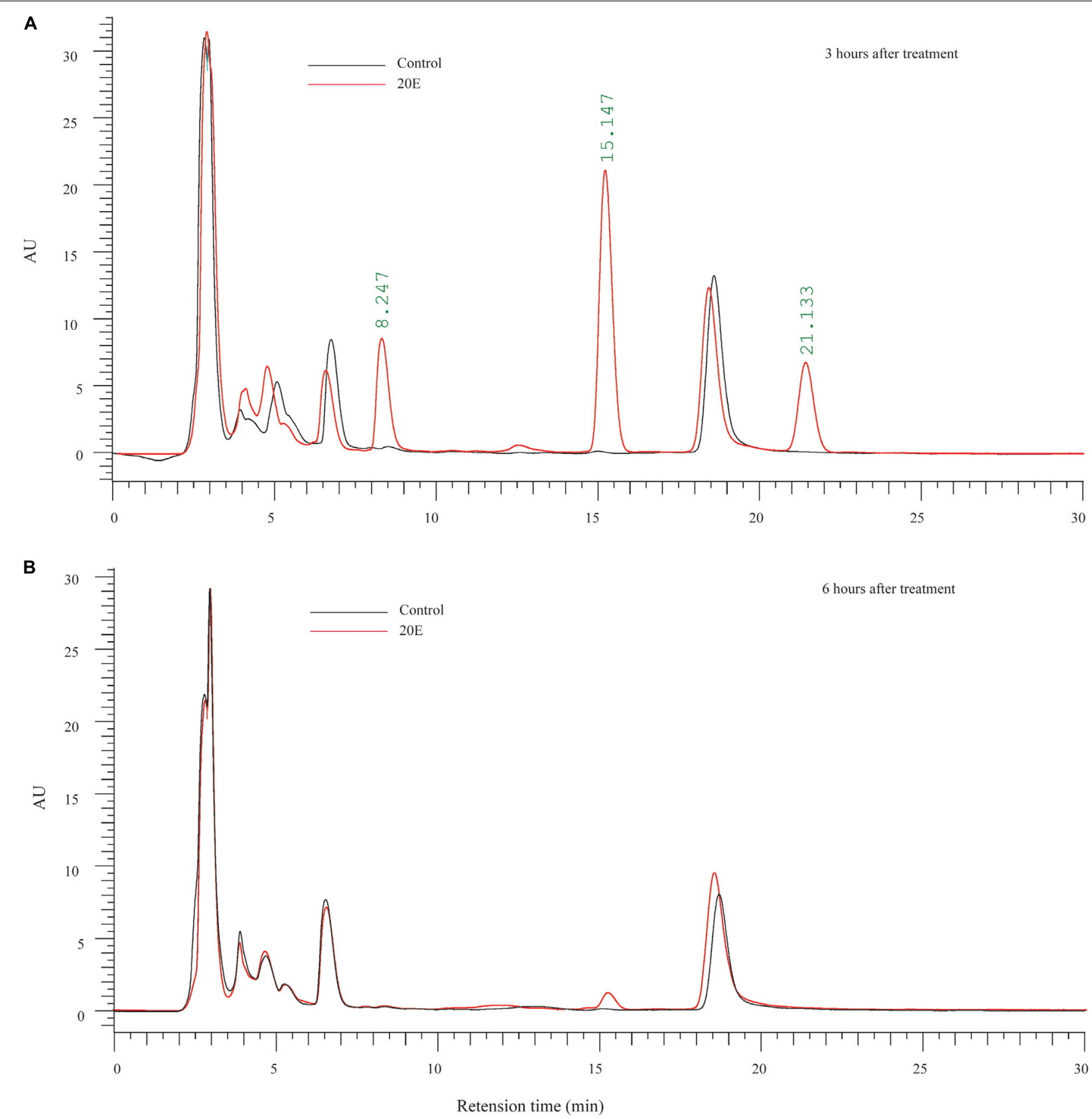

FIGURE 2 | Metabolic fate of ingested 20E. (A) HPLC analysis of ecdysteroids extracted from the feces of larvae at $3 \mathrm{~h}$ after control or 20E treatment. (B) HPLC analysis of ecdysteroids extracted from the feces of larvae at $6 \mathrm{~h}$ after control or $20 \mathrm{E}$ treatment. Black lines represent the extraction from control treated larvae, and red lines represent the extraction from $20 \mathrm{E}$ treated larvae.

Figure S1). According to the mass spectra shown in previous analysis, the SAP and TAP are the 20E-22-oleate and 20E22 -stearate, respectively (Kubo et al., 1987). In addition, the amount of the 20E and metabolites from feces of each larva is calculated. At $3 \mathrm{~h}$ after hormone treatment, $3.834 \pm 0.145 \mu \mathrm{g}$ $20 \mathrm{E}, 8.810 \pm 0.553 \mu \mathrm{g} 20 \mathrm{E}-22$-oleate, and $2.875 \pm 0.245 \mu \mathrm{g}$ $20 \mathrm{E}-22$-stearate were detected. This result indicated about $72.34 \%$ ingested 20E were excreted in feces in the form of $20 \mathrm{E}(19.17 \%)$ and 22-acyl esters (58.43\%). Moreover, $6 \mathrm{~h}$ after treatment, 20E and 20E-22-stearate peaks disappeared, and only a small peak of 20E-22-oleate could be detected (Figure 2B). We also detected minor amounts of 20E-22-oleate and 20E-22-stearate in the hemolymph and fat body (Supplementary Figure S2), indicating ecdysteroid 22-acyl esters can pass through the gut and store in other tissues.

Taken together, our results indicated that the high resistance to $20 \mathrm{E}$ is contributed to by the transformation into lipophilic ecdysteroid esters. 


\section{Transcriptomic Analysis}

As shown above, exogenous $20 \mathrm{E}$ has no effect on the normal growth and development of the $H$. armigera larva. We further examined whether $20 \mathrm{E}$, an important developmental regulator, had roles of gene expression alteration at the transcriptomic level. Firstly, we identified 930 differently expressed genes (DEG) by comparing the gene expression levels between control and $20 \mathrm{E}$ treated larval midgut. Among them, 448 genes were significantly up-regulated by $20 \mathrm{E}$, and 482 genes were decreased (Figure 3A). Compared with the down-regulated genes, the functional GO terms of up-regulated genes were mainly enriched in molecular regulation and signal transduction (Figure 3B). As the important steroid hormone, 20E could elicit its signal transduction and affect the expression change of several genes. In the up-regulated gene dataset, we identified 15 transcription factors. Almost half of those TFs (7/15) are known 20E response genes, such as ecdysone receptor $(\mathrm{EcR})$, broad-complex, and E75 (Supplementary Table S2). Among them, hormone receptor 3 (HR3), which is considered as a central regulator in 20E-driven developmental transitions, showed the largest expressional change after 20E treatment (Lam et al., 1997). Furthermore, KEGG analysis was performed. Down regulated genes were enriched in insect hormone biosynthesis, lysosome, and metabolism (Figure 3C). For the up-regulated genes, the metabolic pathway, biosynthesis of secondary metabolites, and some detoxing related cytochrome $\mathrm{P} 450$ were enriched in KEGG analysis, indicating the cotton bollworm may promote its detoxing system to conquer the exogenous $20 \mathrm{E}$.

In addition, the feeding test shown above indicates that $20 \mathrm{E}$ has no detrimental effects on the growth and nutrition conversion of the larvae. Therefore, we checked the expression of several digestive enzyme genes. The main digestive enzymes of insects are carbohydrases ( $\beta$-glucosidase, $\alpha$-amylase, trehalase, $\alpha$-glucosidase, and $\beta$-galactosidase), lipases, and proteinases (trypsin and aminopeptidase) (Terra and Ferreira, 2012). From midgut transcriptomic data, we identified several genes (Table 1). Compared with control, the transcript level of most of the digestive enzyme genes were not significantly changed after $20 \mathrm{E}$ treatment (Log2(fold change) $>2$ ). This result further supports that the cotton bollworm can tolerate high concentrations of $20 \mathrm{E}$ without altering its digestion ability.

\section{Identification and Expression of Candidate Genes}

Previous studies showed that the expression of ecdysone degradation enzymes, such as ecdysone oxidase and Cyp18a1, could be induced by ecdysteroids (Li et al., 2014; Sun et al., 2017). Therefore, we focused on the $20 \mathrm{E}$ up-regulated genes from transcriptome data to identify the candidate enzymes contributing to the high ecdysteroid resistance of the cotton bollworm. Firstly, we used the Venn diagram between the top 50 most differentially expressed genes and top 50 most highly expressed genes in $20 \mathrm{E}$ treatment sample to filter the target genes (Figures 3A1,A2). Five genes were detected in the intersection of the two datasets. Interestingly, four of five genes were annotated as enzymes including three cytochrome
P450 genes (cyp6b2, cyp18a1, and cyp18b1) and a long-chainfatty-acid-CoA ligase (Long-FACL). Among them, the function of one cytochrome $\mathrm{P} 450$ gene annotated as cyp6b2 is still unknown. Cyp18a1 is served as 26-hydroxylase and mediates the inactivation of the ecdysteroids (Rewitz et al., 2010; Guittard et al., 2011). Cyp18a1 has been identified in different insects, while as its paralog gene - cyp18b1 is only reported in Bombyx mori (Li et al., 2014). To trace the evolution of cyp18 genes, we attempted to search for more cyp18 genes in other insects. As a result, cyp18a1 was widely distributed in different insects including Lepidoptera, Diptera, Coleoptera, and Hymenoptera. Interestingly, cyp $18 \mathrm{~b} 1$ was only detected in lepidopteran insects. Furthermore, phylogeny analysis showed cyp18al proteins from different species were clustered together, and cyp18b1 proteins formed a single clade (Supplementary Figure S3). This result indicates cyp18a1 underwent lineage specific gene duplication after the Lepidoptera-other taxa split generating its paralog-cyp18b1.

For the Long-FACL, the enzyme plays key roles in various metabolic and regulatory processes by catalyzing the formation of fatty acyl-CoA (Hisanaga et al., 2004). More importantly, fatty acyl-CoA was reported as the co-substrates (acyl-group donor) for the ecdysteroid-22-O-acyltransferase activity (Kubo et al., 1987, 1994; Zhang and Kubo, 1992a,b). Therefore, we thought Long-FACL may take part in the ecdysteroids esterification pathway.

Ecdysteroid-22-O-acyltransferase belongs to the family of membrane-bound $O$-acyltransferase (MBOAT; Zhang and Kubo, 1992a,b; Kubo et al., 1994). From the transcriptome data, only four MBOAT genes had a transcription signal, and none of them were differently expressed between control and $20 \mathrm{E}$ treatment (Supplementary Table S3). In addition, according to previous studies, ecdysteroid-22-O-acyltransferase is located on the plasma membrane of the gut epithelial cells (Kubo et al., 1994). Therefore, based on the subcellular localization, transcript level, and annotation, MSTRG.8235 (Sterol O-acyltransferase, SATF) was considered as potential candidate of the ecdysteroid22-O-acyltransferase (Supplementary Table S3).

In addition, we performed real-time PCR to confirm the RNA-seq data and surveyed the expression pattern of the genes identified above. The expression pattern of those genes by qRTPCR was consistent with the transcriptomic changes of RNA-seq data (Figure 4). In order to further examine the effect of $20 \mathrm{E}$ on gene expression, more time points after the hormone treatment were chosen. All genes, excluding SATF, could be quickly induced by $20 \mathrm{E}$ from $1 \mathrm{~h}$ after treatment, and reached expression peaks at $3 \mathrm{~h}$ after treatment. Moreover, for three cytochrome P450 genes, the induction mediated by $20 \mathrm{E}$ was detected in different tissues, such as head, fat body, and epidermis. However, LongFACL was only induced in the midgut. Meanwhile, we surveyed the $5^{\prime}$ upstream of the Long-FACL gene and identified several $20 \mathrm{E}$ related cis-regulatory sites by bioinformatics prediction ${ }^{4}$, such as EcR/ultraspiracle (USP), broad-complex (Br-C), and E74, further indicating it is a $20 \mathrm{E}$ inducible gene (Supplementary Figure S4). For SATF gene, the gene showed the constant and

\footnotetext{
${ }^{4}$ http://jaspar.genereg.net/
} 
A

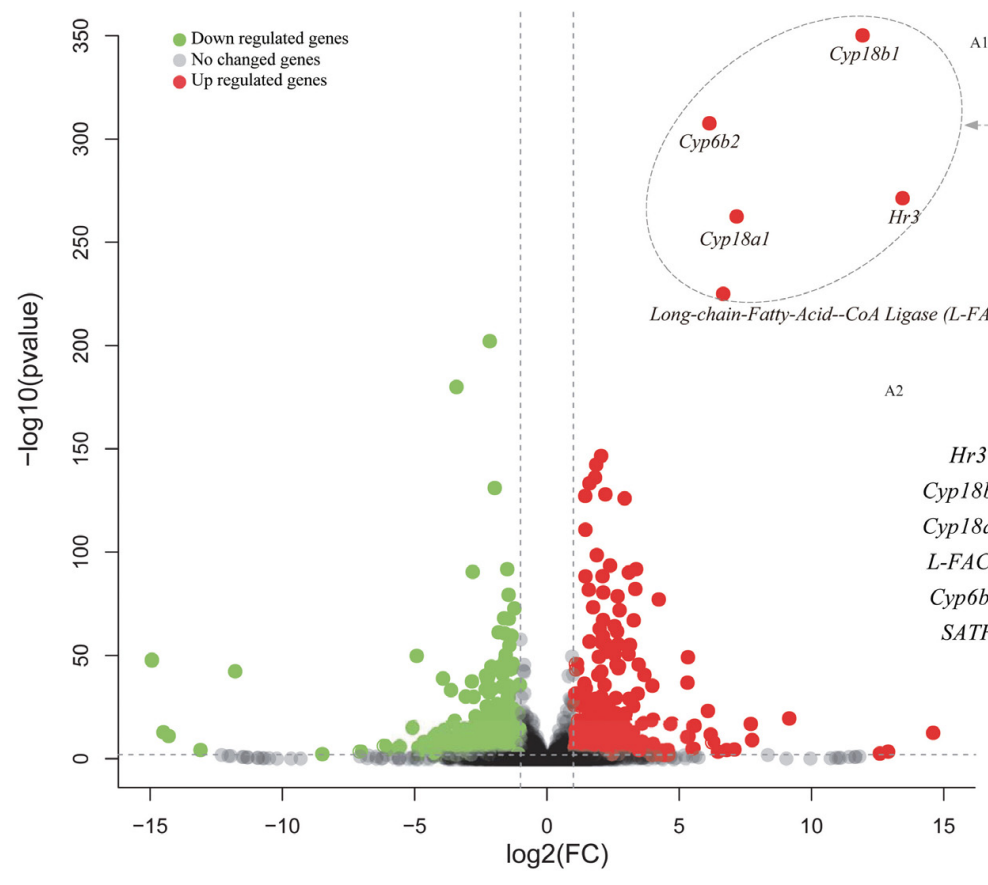

B

Level2 Go terms of out

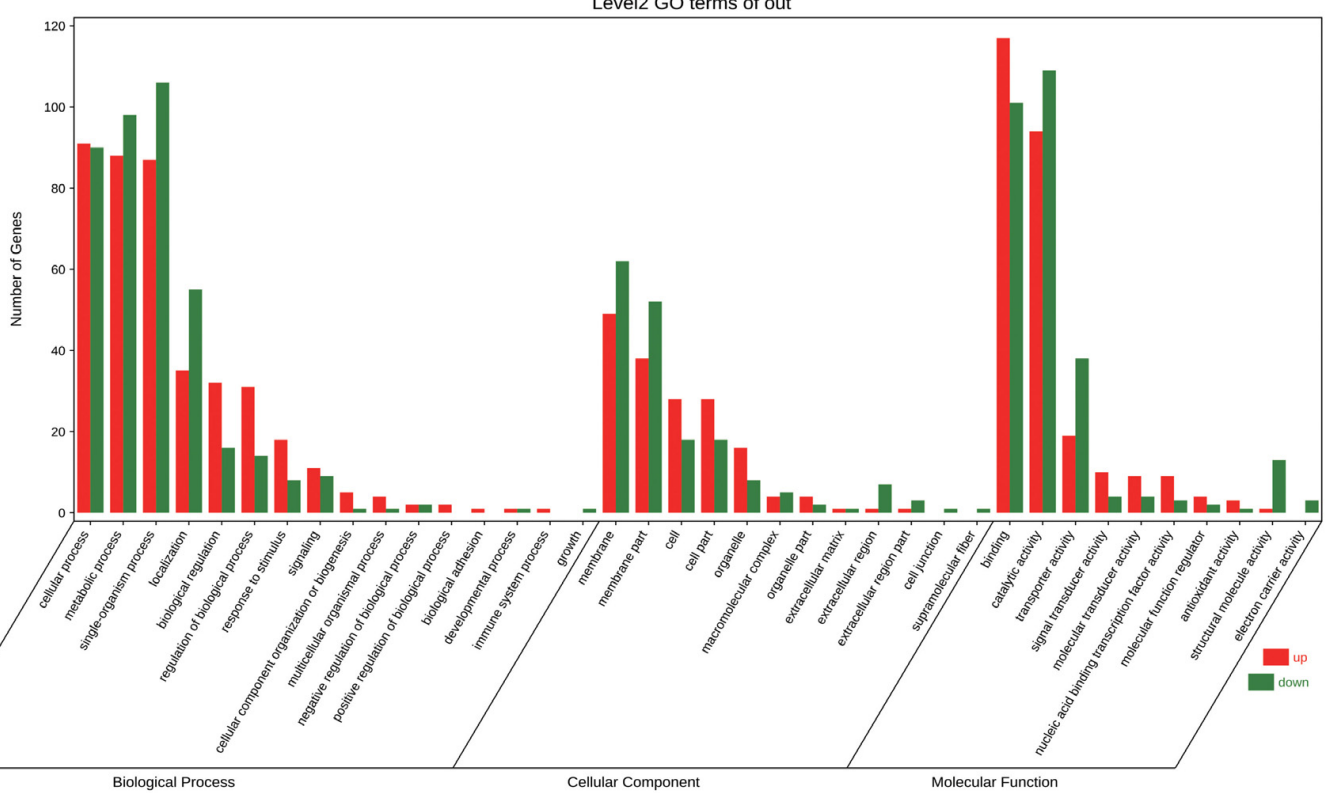

C

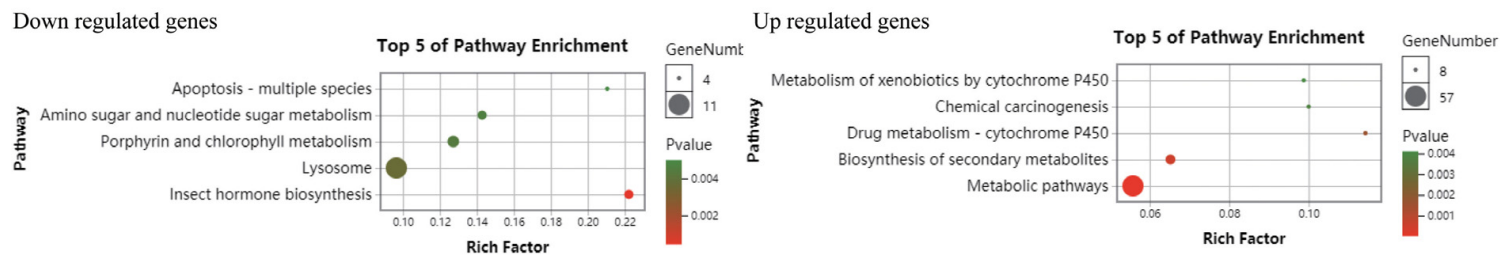

FIGURE 3 | Comparative transcriptome analysis of the larvae midgut after control and 20E treatment. (A) Volcano plot of the fold change of transcripts in $20 \mathrm{E}$ treated larvae midgut compared to control; Inner figure A1 represents Venn diagram between top 50 most differentially expressed genes and top 50 most highly expressed genes in 20E treatment sample; Inner figure A2 represents the TPM value of the candidate genes identified by Venn diagram; TPM means transcripts per million. (B) Gene ontology analysis of the differently expressed genes between control and 20E treated sample. (C) KEGG analysis of the differently expressed genes between control and $20 \mathrm{E}$ treated sample. 
TABLE 1 | Identification and expression of genes encoding digestive enzymes.

\begin{tabular}{|c|c|c|c|c|c|c|}
\hline Classification & Gene ID & AVG. TPM-Control & AVG. TPM-20E & $\log 2(f c)$ & FDR & Annotation \\
\hline \multirow[t]{10}{*}{ Carbohydrases } & MSTRG.11222 & 603.015 & 677.625 & 0.168293 & 0.175097 & $\alpha$-Glucosidase \\
\hline & MSTRG.1618 & 36.655 & 45.505 & 0.312015 & 0.58751 & $\alpha$-Glucosidase \\
\hline & MSTRG.9541 & 59.56 & 57.915 & -0.04041 & 1 & $\alpha$-Glucosidase \\
\hline & MSTRG.8235 & 20.44 & 20.67 & 0.016143 & 1 & $\alpha$-Glucosidase \\
\hline & MSTRG.11087 & 558.86 & 387.52 & -0.52822 & $1.29 E-08$ & $\alpha$-Amylase \\
\hline & MSTRG.11086 & 1737.805 & 1669.605 & -0.05776 & 0.903124 & $\alpha$-Amylase \\
\hline & MSTRG.9468 & 2387.025 & 2526.855 & 0.082129 & 0.4770584 & $\alpha$-Amylase \\
\hline & MSTRG.9467 & 78.925 & 80.155 & 0.02231 & 1 & $\alpha$-Amylase \\
\hline & MSTRG.990 & 44.175 & 33.755 & -0.38813 & 0.4591 & Trehalase \\
\hline & MSTRG.2686 & 3.885 & 5.2 & 0.420597 & 1 & $\beta$-Galactosidase \\
\hline \multirow[t]{3}{*}{ Lipases } & MSTRG.1592 & 2373.865 & 2119.01 & -0.16385 & 0.0704606 & Lipase \\
\hline & MSTRG.1587 & 32.05 & 30.34 & -0.0791 & 1 & Lipase \\
\hline & MSTRG.1594 & 29.765 & 4.79 & -2.63552 & $1.82 E-08$ & Lipase \\
\hline \multirow[t]{5}{*}{ Proteinases } & MSTRG.1715 & 22.44 & 11.905 & -0.91451 & 0.089117 & Tyrpsin \\
\hline & MSTRG.10422 & 1037.475 & 827.915 & -0.32552 & 0.0006072 & Aminopeptidase N \\
\hline & MSTRG.10425 & 1095.79 & 1414.215 & 0.36803 & $1.84 E-05$ & Aminopeptidase N \\
\hline & MSTRG.10428 & 1124.28 & 735.375 & -0.61245 & $4.52 E-13$ & Membrane alanyl aminopeptidase \\
\hline & MSTRG.10429 & 788.08 & 619.355 & -0.34758 & 0.0001199 & Membrane alanyl aminopeptidase \\
\hline
\end{tabular}

obvious expression in the midgut, and steroid hormone had no effect on its transcript.

\section{Heterologous Expression and Enzyme Assay}

In order to examine the enzyme activity of two functional unknown enzymes (Cyp6b2 and SATF), the cotton bollworm genes were expressed in Drosophila S2 cell. Using western blotting by Flag antibody, one specific band corresponding to the molecular masses of 55 and $60 \mathrm{kDa}$ was detected in the lysis of pMT-SATF and pMT-Cyp6b2 plasmid transfected cells, respectively (Supplementary Figure S5). This result indicated that both genes were successfully expressed in $S 2$ cell. Thereafter, the crude enzyme solutions were used to measure their activity. As shown in the reversed-phase HPLC UV chromatograms, compared to the control (pMT plasmid transfected cell), crude enzymes from SATF transfected cell can covert 20E into its 22oleate-acyl ester form (Figure 5). It should be noted that this transformation can only be detected with the supplement of oleoyl-CoA (Figure 5). These data demonstrate that SATF codes for an ecdysteroid-22-O-acyltransferase.

For cyp 662 gene, HPLC showed a similar peak profile with control, indicating the enzyme cannot convert 20E (data not shown).

\section{DISCUSSION}

A total of $45 \%$ of insect species feed on plants (Schoonhoven et al., 2005). Plants do not just passively tolerate when suffering from phytophagy insects, instead, they evolved some elegant mechanisms to minimize or inhibit the hazard that insects impose on them. 20-hydroxyecdysone, an essential developmental regulator in insects, is also a major phytoecdysteroids in plants. Therefore, the phytoecdysteroid is considered as defense to negatively affect herbivore growth and survival. In parallel, some insects also develop strategies to overcome the defensive system. In this study, we found that $H$. armigera is remarkably tolerate to high concentrations of ingested exogenous $20 \mathrm{E}$ (up to $50 \mu \mathrm{g} / \mathrm{larva}$ ) without any adverse effects on food consumption and development. Correspondingly, midgut transcriptome analysis also showed most of the digestive enzyme genes kept the same stable expression pattern as the control. This phenomenon maybe resulted from the high efficiency to quickly transform and excrete the ingested $20 \mathrm{E}$ within a few hours. In Heliothis virescens, Zhang and Kubo (1993) also found over 70\% of radioactivity was recovered in feces at $1 \mathrm{~h}$ after oral treatment of ${ }^{[3 \mathrm{H}]}$ ecdysone (Zhang and Kubo, 1993). Those results indicated some noctuid larvae have a high rate of excretion system to detoxify exogenous hormones.

The midgut is the main tissue for insects to detoxify the plant allelochemicals (Despres et al., 2007). In order to identify the candidate genes contributing to ecdysteroid tolerance of the cotton bollworm, we focused on the $20 \mathrm{E}$ induced genes in the midgut transcriptomic data. Several previous studies have demonstrated that some ecdysone degradation pathways, including 3-epimerization and 26-hydroxylation, also were induced by the hormone (Li et al., 2014; Sun et al., 2017). Hence, by comparative transcriptome analysis, we found four genes encoding three cytochrome P450 enzymes (cyp6b2, cyp18a1, and cyp18b1) and one Long-FACL. Cytochrome P450 enzymes are well known for their roles in the metabolism of insecticide and plant secondary compounds (Heidel-Fischer and Vogel, 2015). Cyp18a1 and Cyp18b1 serve as 26-hydroxylase to convert 20E into 20-hydroxyecdysonoic acid (Rewitz et al., 2010; Guittard et al., 2011). However, we cannot identify their product (20hydroxyecdysonoic acid) in the cotton bollworm feces. In H. virescens, Zhang and Kubo (1993) only detected trace amounts of 20-hydroxyecdysonoic acid in the feces of the larvae orally injected by $20 \mathrm{E}$ (Zhang and Kubo, 1993). It should be pointed 

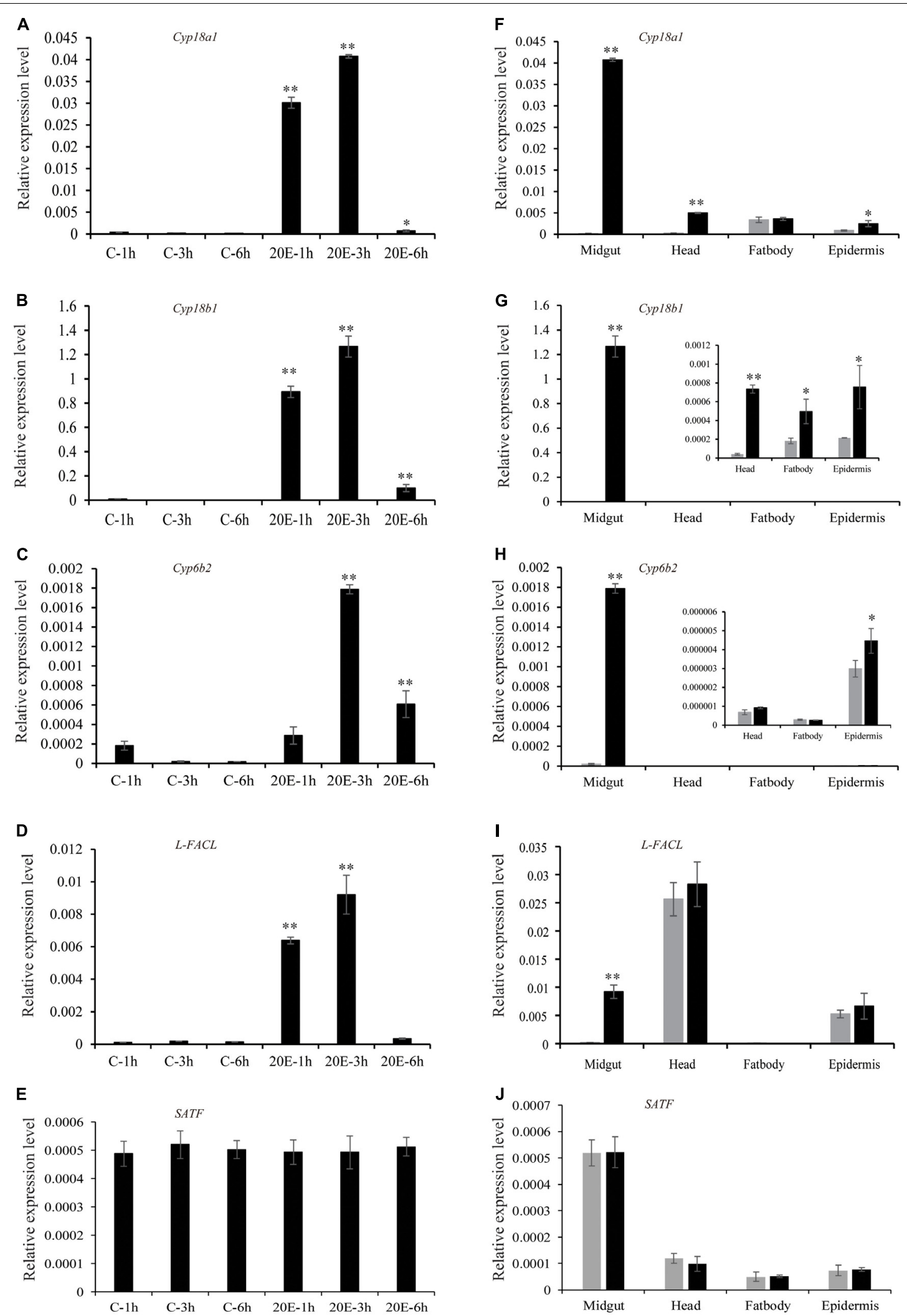

FIGURE 4 | Expression pattern of the candidate genes after 20E treatment. (A-E) The expression of the candidate genes in cotton bollworm larvae midgut at different time points after control or $20 \mathrm{E}$ treatment. (F-J) The expression of the candidate genes in different tissues at $3 \mathrm{~h}$ after control or $20 \mathrm{E}$ treatment. ${ }^{\star} p$ value via student's $t$-test based on three replicates $\left({ }^{*} p<0.05 ;{ }^{* \star} p<0.01\right)$. 

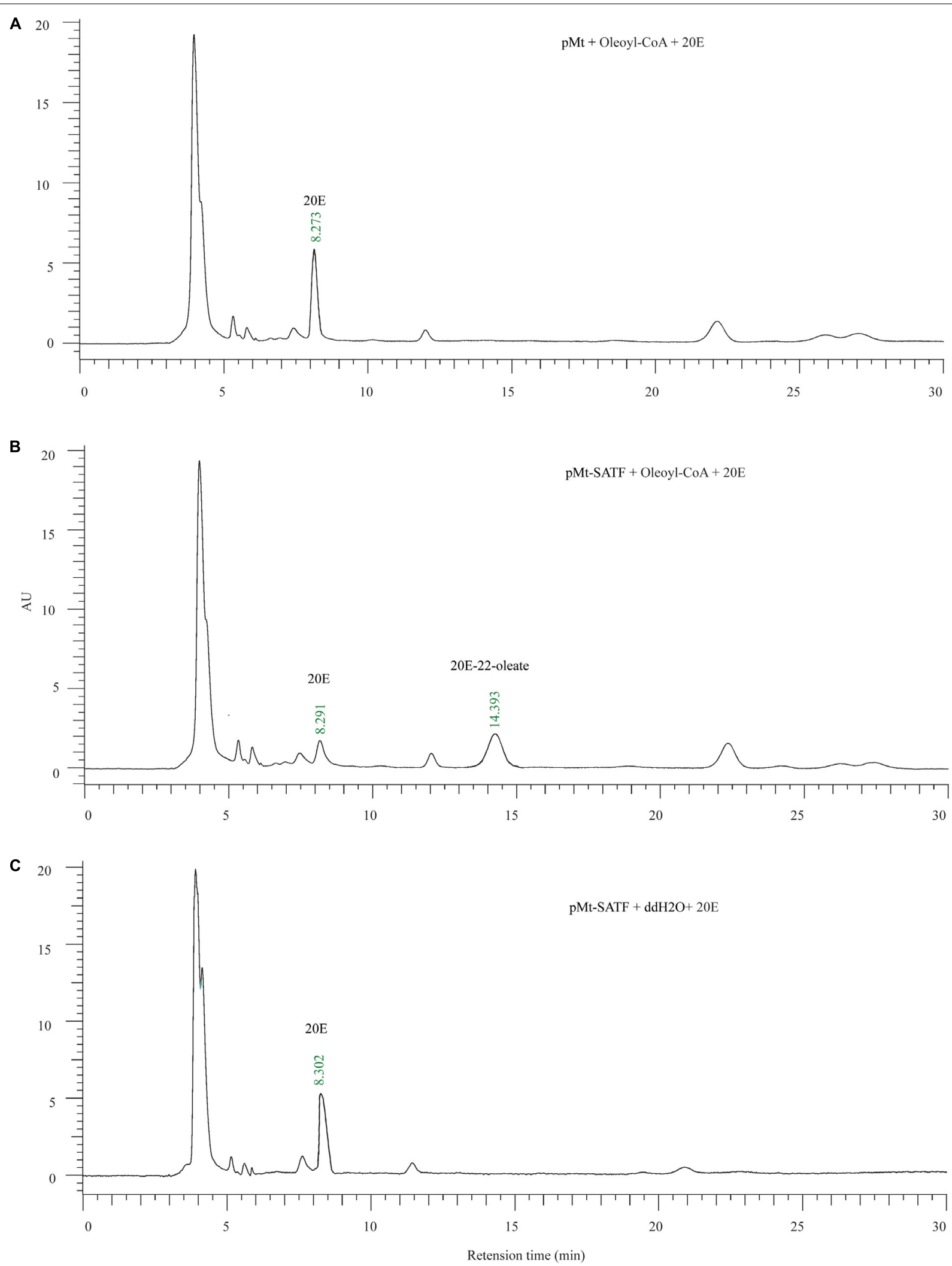

FIGURE 5 | Enzymic activity of recombinant Sterol O-acyltransferase, SATF proteins. (A) The enzyme assay of the supernatant of the control vector (pMt) infected cell with the 20E and Oleoyl-CoA. (B) The enzyme assay of the supernatant of the pMt-SATF infected cell with the 20E and Oleoyl-CoA. (C) The enzyme assay of the supernatant of the pMt-SATF infected cell with the $20 \mathrm{E}$ and water. 
out that though Cyp18a1 and Cyp18b1 genes were highly induced after $20 \mathrm{E}$ treatment, our research and previously published papers proved the majority of ingested 20E were converted into acyl-ester forms, indicating the ecdysteroid esterification pathway is the key pathway for detoxifying exogenous hormones (Robinson et al., 1987; Zhang and Kubo, 1993). Hence, the function of the 26-hydroxylases needs further investigation in the future.

In the normal feeding stage of $H$. virescens, the midgut crude enzyme extract showed ecdysteroid-22-O-acyltransferase activity only with the supplement of fatty acyl-CoA (Zhang and Kubo, 1992b; Kubo et al., 1994). Those results give us two important hints: (1) the larvae midgut itself contains the acyltransferase under normal conditions and (2) the enzyme activity is fatty acyl-CoA dependent. Indeed, the SATF, which was proven as ecdysteroid-22-O-acyltransferase in this study, showed similar expression levels between control and 20E treatment, and the gene was mainly expressed in thr digestive system. In the $H$. virescens midgut, enzyme activity of the acyltransferase also had no change after 20E incubation (Zhang and Kubo, 1992b). However, the transcript level of Long-FACL encoding an enzyme to produce fatty acyl-CoA is quite low in the control midgut but can be highly and quickly induced by 20E. As an important regulator, ecdysone firstly binds to the EcR/USP complex and subsequently induces several primary and late response transcription factors (Thummel, 2001). We detected several 20E related cis-regulatory sites, such as EcR/USP, Br-C, and E74, located on the $5^{\prime}$ upstream of the Long-FACL gene indicating it is a $20 \mathrm{E}$ inducible gene in the midgut. In total, it would appear that the insect only starts to esterify exogenous ecdysteroid in the presence of acyl esters supplied by a $20 \mathrm{E}$ inducible enzyme Long-FACL.

Blackford and Dinan (1997a) proposed that the sensitivity of insects to phytoecdysteroids is correlated to their feeding habits (monophagous insects: sensitive; oligophagous insects: semitolerant; polyphagous insects: tolerant) (Blackford and Dinan, 1997a). Our recent results partly explain how a polyphagous insect, the cotton bollworm, utilizes esterification pathway to degrade the exogenous ecdysteroid to conquer the plant defense. However, the genes involved in this pathway are also conserved in lepidopteran insects including $B$. mori, a wellknown monophagous and 20E sensitive insect (Kubo et al., 1983). It is an interesting question why domesticated silkworm cannot protect themselves against $20 \mathrm{E}$ through the same enzymes as the cotton bollworm. In addition, ecdysteroid-22-acylesters are also found in the ovaries and eggs of some insects,

\section{REFERENCES}

Adler, J. H., and Grebenok, R. J. (1999). Occurrence, biosynthesis, and putative role of ecdysteroids in plants. Crit. Rev. Biochem. Mol. Biol. 34, 253-264. doi: $10.1080 / 10409239991209282$

Aly, R., Ravid, U., Abu-Nassar, J., Botnick, I., Lebedev, G., Gal, S., et al. (2011). Biological activity of natural phytoecdysteroids from Ajuga iva against the sweetpotato whitefly Bemisia tabaci and the persea mite Oligonychus perseae. Pest. Manag. Sci. 67, 1493-1498. doi: 10.1002/ps.2203 and the hormone derivates are served as storage forms for embryogenesis (Connat et al., 1988; Whiting and Dinan, 1989). Thus, more studies are needed to survey the novel function of the ecdysteroid esterification pathway during insect developmental process in the future.

\section{DATA AVAILABILITY STATEMENT}

The raw data of RNA-seq has been submitted to NCBI (SRA accession: PRJNA588578).

\section{ETHICS STATEMENT}

Experiments were conducted in accordance with the protocol approved by the Institutional Animal Care and Use Committee of the Chongqing University (permit number CBE-A201607020).

\section{AUTHOR CONTRIBUTIONS}

WS and $Z Z$ conceived and designed the experiments. HD, XY, $\mathrm{ZB}$, and $\mathrm{XL}$ performed the experiments. $\mathrm{HD}$ and $\mathrm{XY}$ analyzed the data. ZZ and WS contributed reagents, materials, and analysis tools. HD and WS wrote the manuscript.

\section{FUNDING}

This work was supported by the funded by Chongqing Research Program of Basic Research and Frontier Technology (No. cstc2017jcyjAX0413), and the Fundamental Research Funds for the Central Universities (No. 2019CDXYSM0017).

\section{ACKNOWLEDGMENTS}

We thank all other members of Zhang's lab for their laboratory assistance.

\section{SUPPLEMENTARY MATERIAL}

The Supplementary Material for this article can be found online at: https://www.frontiersin.org/articles/10.3389/fphys. 2020.00508/full\#supplementary-material

Anders, S., Pyl, P. T., and Huber, W. (2015). HTSeq-a Python framework to work with high-throughput sequencing data. Bioinformatics 31, 166-169. doi: 10.1093/bioinformatics/btu638

Blackford, M., Clarke, B., and Dinan, L. (1996). Tolerance of the Egyptian cotton leafworm Spodoptera littoralis (Lepidoptera: Noctuidae) to ingested phytoecdysteroids. J. Insect Physiol. 42, 931-936. doi: 10.1016/0022-1910(96) 00052-2

Blackford, M., and Dinan, L. (1997a). The effects of ingested 20-hydroxyecdysone on the larvae of Aglais urticae, Inachis io, Cynthia cardui (Lepidoptera: 
Nymphalidae) and Tyria jacobaeae (Lepidoptera: Arctiidae). J. Insect Physiol. 43, 315-327. doi: 10.1016/s0022-1910(96)00112-6

Blackford, M., and Dinan, L. (1997b). The effects of ingested ecdysteroid agonists (20-hydroxyecdysone, RH5849 and RH5992) and an ecdysteroid antagonist (cucurbitacin B) on larval development of two polyphagous lepidopterans (Acherontia atropos and Lacanobia oleracea). Entomol. Exp. Appl. 83, 263-276. doi: 10.1046/j.1570-7458.1997.00181.x

Blackford, M. J., Clarke, B. S., and Dinan, L. (1997). Distribution and metabolism of exogenous ecdysteroids in the Egyptian cotton leafworm Spodoptera littoralis (Lepidoptera: Noctuidae). Arch. Insect. Biochem. Physiol. 34, 329-346. doi: 10.1002/(sici) 1520-6327(1997)34:3<329::aid-arch7>3.0.co;2-p

Butenandt, A., and Karlson, P. (1954). Über die isolierung eines metamorphosehormons der insekten in kristallisierter form. Zeitschrift Naturforschung B 9, 389-391. doi: 10.1515/znb-1954-0601

Chaubey, M. K. (2018). Role of phytoecdysteroids in insect pest management: a review. J. Agron. 17, 1-10. doi: 10.3923/ja.2018.1.10

Chen, C., Xia, R., Chen, H., and He, Y. (2018). TBtools, a Toolkit for Biologists integrating various biological data handling tools with a user-friendly interface. BioRxiv [Preprint] doi: 10.1101/289660

Connat, J. L., Dotson, E. M., and Diehl, P. A. (1988). Apolar conjugates of ecdysteroids are not used as a storage form of molting hormone in the argasid tick Ornithodoros moubata. Arch. Insect. Biochem. Physiol. 9, 221-235. doi: 10.1002/arch.940090306

Despres, L., David, J.-P., and Gallet, C. (2007). The evolutionary ecology of insect resistance to plant chemicals. Trends Ecol. Evol. 22, 298-307. doi: 10.1016/j.tree. 2007.02.010

Dinan, L. (2001). Phytoecdysteroids: biological aspects. Phytochemistry 57, $325-$ 339. doi: 10.1016/s0031-9422(01)00078-74

Götz, S., García-Gómez, J. M., Terol, J., Williams, T. D., Nagaraj, S. H., Nueda, M. J., et al. (2008). High-throughput functional annotation and data mining with the Blast2GO suite. Nucl Acid Res. 36, 3420-3435. doi: 10.1093/nar/ gkn176

Guittard, E., Blais, C., Maria, A., Parvy, J.-P., Pasricha, S., Lumb, C., et al. (2011). CYP18A1, a key enzyme of Drosophila steroid hormone inactivation, is essential for metamorphosis. Dev. Biol. 349, 35-45. doi: 10.1016/j.ydbio.2010. 09.023

Heidel-Fischer, H. M., and Vogel, H. (2015). Molecular mechanisms of insect adaptation to plant secondary compounds. Curr. Opin. Insect Sci. 8, 8-14. doi: 10.1016/j.cois.2015.02.004

Hisanaga, Y., Ago, H., Nakagawa, N., Hamada, K., Ida, K., Yamamoto, M., et al. (2004). Structural basis of the substrate-specific two-step catalysis of long chain fatty acyl-CoA synthetase dimer. J. Biol. Chem. 279, 31717-31726. doi: 10.1074/ jbc.m400100200

Jurenka, R., Russell, K., and O’Neal, M. (2017). Phytoecdysteroids as antifeedants towards several beetles that include polyphagous and monophagous feeding guilds. Pest. Manag. Sci. 73, 1633-1637. doi: 10.1002/ps. 4500

Kanehisa, M., and Goto, S. (2000). KEGG: kyoto encyclopedia of genes and genomes. Nucl Acid Res. 28, 27-30.

Kang, X.-L., Zhang, J.-Y., Wang, D., Zhao, Y.-M., Han, X.-L., Wang, J.-X., et al. (2019). The steroid hormone 20-hydroxyecdysone binds to dopamine receptor to repress lepidopteran insect feeding and promote pupation. PLoS Genet. 15:e1008331. doi: 10.1371/journal.pgen.1008331

Kubo, I., Klocke, J. A., and Asano, S. (1981). Insect ecdysis inhibitors from the East African medicinal plant Ajuga remota (Labiatae). Agr. Biol. Chem. 45, 1925-1927. doi: 10.1271/bbb1961.45.1925

Kubo, I., Klocke, J. A., and Asano, S. (1983). Effects of ingested phytoecdysteroids on the growth and development of two lepidopterous larvae. J. Insect Physiol. 29, 307-316. doi: 10.1016/0022-1910(83)90031-8

Kubo, I., Komatsu, S., Asaka, Y., and de Boer, G. (1987). Isolation and identification of apolar metabolites of ingested 20-hydroxyecdysone in frass of Heliothis virescens larvae. J. Chem. Ecol. 13, 785-794. doi: 10.1007/bf0102 0160

Kubo, I., Zhang, M., De Boer, G., and Uchima, K. (1994). Location of ecdysteroid 22-O-acyltransferase in the larvae of Heliothis virescens. Entomol. Exp. Appl. 70, 263-272. doi: 10.1111/j.1570-7458.1994.tb00755.x
Lafont, R., and Horn, D. (1989). "Phytoecdysteroids: structures and occurrence," in Ecdysone From Chemistry to Mode of Action, ed. J. Koolman (New York, NY: Thieme Medical Publishers), 39-64.

Lam, G. T., Jiang, C., and Thummel, C. S. (1997). Coordination of larval and prepupal gene expression by the DHR3 orphan receptor during Drosophila metamorphosis. Development 124, 1757-1769.

Li, Z., Ge, X., Ling, L., Zeng, B., Xu, J., Aslam, A. F., et al. (2014). CYP18A1 regulates tissue-specific steroid hormone inactivation in Bombyx mori. Insect Biochem. Mol. Biol. 54, 33-41. doi: 10.1016/j.ibmb.2014.08.007

Love, M. I., Huber, W., and Anders, S. (2014). Moderated estimation of fold change and dispersion for RNA-seq data with DESeq2. Genome Biol. 15: 550.

Martin, T., Ochou, G. O., Djihinto, A., Traore, D., Togola, M., Vassal, J. M., et al. (2005). Controlling an insecticide-resistant bollworm in West Africa. Agr. Ecosyst. Environ. 107, 409-411. doi: 10.1016/j.agee.2004.11.006

Mithöfer, A., and Boland, W. (2012). Plant defense against herbivores: chemical aspects. Annu. Rev. Plant Biol. 63, 431-450. doi: 10.1146/annurev-arplant042110-103854

Mondy, N., Caïssa, C., Pitoizet, N., Delbecque, J. P., and Corio-Costet, M. F. (1997). Effects of the ingestion of Serratula tinctoria extracts, a plant containing phytoecdysteroids, on the development of the vineyard pest Lobesia botrana (Lepidoptera: Tortricidae). Arch. Insect. Biochem. Physiol. 35, 227-235. doi: 10.1002/(sici) 1520-6327(1997)35:1/2<227::aid-arch21 >3.0.co;2-c

Nakanishi, K., Koreeda, M., Sasaki, S., Chang, M., and Hsu, H. (1966). Insect hormones. The structure of ponasterone A, insect-moulting hormone from the leaves of Podocarpus nakaii Hay. Chem. Comm. 24, 915-917.

Pertea, M., Kim, D., Pertea, G. M., Leek, J. T., and Salzberg, S. L. (2016). Transcriptlevel expression analysis of RNA-seq experiments with HISAT, StringTie and Ballgown. Nat. Prot. 11:1650. doi: 10.1038/nprot.2016.095

Rewitz, K. F., Yamanaka, N., and O'Connor, M. B. (2010). Steroid hormone inactivation is required during the juvenile-adult transition in Drosophila. Dev. Cell 19, 895-902. doi: 10.1016/j.devcel.2010.10.021

Robinson, P., Morgan, E., Wilson, I., and Lafont, R. (1987). The metabolism of ingested and injected $[3 \mathrm{H}]$ ecdysone by final instar larvae of Heliothis armigera. Physiol. Entomol. 12, 321-330. doi: 10.1111/j.1365-3032.1987.tb00757.x

Schmelz, E. A., Grebenok, R. J., Ohnmeiss, T. E., and Bowers, W. S. (2002). Interactions between Spinacia oleracea and Bradysia impatiens: a role for phytoecdysteroids. Arch. Insect. Biochem. Physiol. 51, 204-221. doi: 10.1002/ arch.10062

Schoonhoven, L. M., Van Loon, B., van Loon, J. J., and Dicke, M. (2005). InsectPlant Biology. Oxford: Oxford University Press on Demand.

Soriano, I. R., Riley, I. T., Potter, M. J., and Bowers, W. S. (2004). Phytoecdysteroids: a novel defense against plant-parasitic nematodes. J. Chem. Ecol. 30, 1885-1899. doi: 10.1023/b:joec.0000045584.56515.11

Sun, W., Shen, Y. H., Qi, D. W., Xiang, Z. H., and Zhang, Z. (2012). Molecular cloning and characterization of Ecdysone oxidase and 3-dehydroecdysone-3 $\alpha$ reductase involved in the ecdysone inactivation pathway of silkworm, Bombyx mori. Int. J. Biol. Sci. 8:125. doi: 10.7150/ijbs.8.125

Sun, W., Wang, C. F., and Zhang, Z. (2017). Transcription factor E74A affects the ecdysone titer by regulating the expression of the EO gene in the silkworm, Bomby mori. Biochim. Biophys. Acta 1861, 551-558. doi: 10.1016/j.bbagen.2016. 11.017

Tanaka, Y., and Naya, S. (1995). Dietary effect of ecdysone and 20hydroxyecdysone on larval development of two lepidopteran species. Appl. Entomol. Zool. 30, 285-294. doi: 10.1303/aez.30.285

Terra, W. R., and Ferreira, C. (2012). "Biochemistry and molecular biology of digestion," in Insect Molecular Biology and Biochemistry, ed. L. I. Gilbert (London: Elsevier Science Publishers), 365-418. doi: 10.1016/b978-0-12384747-8.10011-x

Thummel, C. S. (2001). Molecular mechanisms of developmental timing in C. elegans and Drosophila. Dev. Cell 1, 453-465. doi: 10.1016/s1534-5807(01) 00060-0

Whiting, P., and Dinan, L. (1989). Identification of the endogenous apolar ecdysteroid conjugates present in newly-laid eggs of the house cricket (Acheta domesticus) as 22-long-chain fatty acyl esters of ecdysone. Insect. Biochem. 19, 759-765. doi: 10.1016/0020-1790(89)90057-7 
Zhang, M., and Kubo, I. (1992a). Characterization of ecdysteroid-22-Oacyltransferase from tobacco budworm, Heliothis virescens. Insect Biochem. Mol. Biol. 22, 599-603. doi: 10.1016/0965-1748(92)90037-f

Zhang, M., and Kubo, I. (1992b). Possible function of ecdysteroid-22O-acyltransferase in the larval gut of tobacco budworm. Heliothis virescens. J. Chem. Ecol. 18, 1139-1149. doi: 10.1007/bf0098 0069

Zhang, M., and Kubo, I. (1993). Metabolic fate of ecdysteroids in larval Bombyx mori and Heliothis virescens. Insect Biochem. Mol. Biol. 23, 831-843. doi: 10. 1016/0965-1748(93)90072-Z
Conflict of Interest: The authors declare that the research was conducted in the absence of any commercial or financial relationships that could be construed as a potential conflict of interest.

Copyright (C) 2020 Duan, Yang, Bu, Li, Zhang and Sun. This is an open-access article distributed under the terms of the Creative Commons Attribution License (CC BY). The use, distribution or reproduction in other forums is permitted, provided the original author(s) and the copyright owner(s) are credited and that the original publication in this journal is cited, in accordance with accepted academic practice. No use, distribution or reproduction is permitted which does not comply with these terms. 